\title{
Representaciones del sujeto-ciudadano en los discursos del "saber experto" en Chile
}

\author{
Juan Sandoval Moya \\ Universidad de Valparaíso, Valparaíso, Chile. E-mail: juan.sandoval@uv.cl
}

\begin{abstract}
Resumen: En el presente artículo se analizan las representaciones del sujeto-ciudadano que se articulan en los discursos sobre la ciudadanía del saber experto chileno. El enfoque metodológico es el estudio de caso y el análisis crítico del discurso, analizando una muestra de documentos institucionales de tres Think Tank de gran influencia nacional: "Instituto Libertad y Desarrollo", "Centro de Estudios Públicos” y "Fundación Chile 21”. El análisis identifica dos posiciones de sujeto-ciudadano en los discursos: el "sujeto post-ciudadano" y el "sujeto neociudadano”, proponiendo como conclusión, que ambas representaciones están articuladas a partir de una racionalidad política común.
\end{abstract}

Palabras clave: Ciudadanía, posición sujeto, think tank, discurso.

\section{Representations of the subject-citizen in the discourse of "expert knowledge" in Chile}

\begin{abstract}
The present paper analyzes the subject-citizen representations articulated in Chilean expert discourse about citizenship. The methodological approach is a case study and a critical analysis of discourse, analyzing a sample of institutional documents of three influential Chilean Think Tanks: "Instituto Libertad y Desarrollo", “Centro de Estudios Públicos” y "Fundación Chile 21”. The result identifies two subject-citizen positions: the "post-citizen subject” and the "neo-citizen subject", concluding that both representations are articulated from a same political rationale
\end{abstract}

Key words: Citizenship, position subject, think tank, discourse

\section{Representações do sujeito-cidadão no discurso do “conhecimento experto" no Chile}

Resumo: O presente artigo analisa as representações do sujeito-cidadão articulado no discurso experto chileno sobre a cidadania. Foram analisados através de um estudo de caso e análise crítica do discurso de uma amostra de documentos institucionais de três influentes Think Tank chilenos_. O analise identifica duas posições de sujeito-cidadão: o “sujeito pós-cidadão” eo “sujeito neo-cidadão", concluindo que ambas as representações são articuladas a partir de uma mesma lógica política

Palavras-chave: Cidadania, posição sujeito, think tank, discurso 


\section{Presentación}

En las sociedades contemporáneas, entre la elite, el espacio mediático y la sociedad civil, emerge una nueva fuente de producción de discursos basada en la cientificidad del saber experto. Las sociedades contemporáneas requieren cada vez más gobernarse a partir del conocimiento reflexivo que pueden tener de sí mismas, de modo que los esfuerzos por medir las características de la vida social se transformen rápidamente en medidas que se toman sobre las propias formas de vida de la sociedad. Tecnologías como las encuestas de opinión, los diagnósticos psicosociales, los estudios de mercado y las prospectivas económicas, constituyen dispositivos de este saber experto, en los cuales se definen las medidas de la sociedad en esta doble acepción: definen cuánto mide la sociedad, al mismo tiempo que estipulan las medidas que se deben tomar para intervenir en ella.

Estas nuevas formas de saber experto toman diversos formatos, por un lado, adquieren un rol supranacional en los estudios sobre los grandes temas de la globalización (migración, sociedad del conocimiento, relaciones internacionales, etc.). Por otro lado, asumen un rol nacional a través del estudio de los grandes temas globales, pero abordándolos en el marco de las lógicas locales del poder y la influencia de los partidos políticos y las instituciones de administración del Estado de cada país (McGann, 2007). En este segundo caso, nos referimos a la red de conocimientos expertos que nutren a los estados nacionales con conocimientos estratégicos y tácticos sobre aquellos que deben ser gobernados, con el objetivo de mejorar su accionar como gobierno. Las instituciones generadoras de estas nuevas formas de saber experto corresponden a los llamados Centros de Estudios o Think Tanks.

Cuando hablamos de Centros de Estudios (o Think Tanks), nos referimos a instituciones de investigación social y análisis estratégico que forman parte de la sociedad civil, constituidas por equipos multidisciplinarios, que ofrecen a los gobiernos y a los parlamentos información técnica sobre asuntos de política nacional e internacional, comercio y seguridad interna y externa. Aunque se definen como instituciones independientes de los gobiernos de turno, y apelan a una suerte de neutralidad técnico-política, a menudo están relacionados con empresas privadas, instituciones académicas, organismos militares, grupos ideológicos o de otro tipo (Garcé, 2009).

Nos interesa analizar los discursos de estos think tanks, porque en la última década, estas instituciones se han transformado en un nuevo actor político en las sociedades desarrolladas y en "vías de desarrollo", toda vez que fundamentándose en la nueva legitimidad que adquiere el manejo de la información en el nuevo orden neocapitalista, han adquirido altísimos niveles de autoridad para racionalizar y fundamentar la toma de decisiones de las elites gobernantes, constituyéndose en un nuevo eslabón que "modula" la relación entre el proceso de toma de decisiones y la legitimidad ciudadana (Weidenbaum, 2010). En Europa, esta tendencia la podemos ver ejemplificada en la influencia que han tenido estas organizaciones en el 
proceso de modernización de los discursos socialdemócratas (Pautz, 2010); o en América Latina, en la influencia que han tenido en la fundamentación de las políticas sociales de los distintos países del continente (Grondona, 2010).

Específicamente en Chile, desde la década de 1990 se institucionalizaron una serie de Centros de Estudios, muchos de ellos continuadores de núcleos de trabajo u organizaciones no gubernamentales (ONG) constituidas en paralelo al Estado y las Universidades durante la Dictadura Militar, pero que a partir del proceso de transición a la democracia se debieron reformular en las nuevas condiciones de la sociedad postdictatorial (Gárate, 2007). El nuevo escenario político llevó a la consolidación de una nueva generación de think tanks, comprometidos con la satisfacción de las necesidades de conocimiento experto de los nuevos actores políticos del país, transformándose en instituciones con una fuerte influencia en el diseño de los programas ideológicos y estratégicos de los partidos políticos y en la administración gubernamental a través del diseño de políticas públicas (Garcé, 2009; Moreno, 2010), constituyéndose en organizaciones fundamentales a la hora de analizar el proceso de construcción de lo social y lo político en el Chile del siglo XXI.

En el presente trabajo nos proponemos estudiar estas instituciones generadoras de saber experto, con el propósito de analizar a través de sus tecnologías de conocimiento, algunos de los discursos hegemónicos que compiten por la construcción social de la ciudadanía. Para el análisis específico, hemos considerado la selección de tres think tanks de gran prestigio intelectual e influencia política en Chile, los cuales representan los nichos intelectuales hegemónicos desde los cuales se construye saber gubernamental, y con su elección se persiguió saturar las tres matrices discursivas que compitieron por la administración de la institucionalidad de Chile en las elecciones Presidenciales del año 2005, a saber, el Instituto Libertad y Desarrollo, el Centro de Estudios Públicos y la Fundación Chile 21.

\section{Marco conceptual}

Nuestro enfoque de estudio tiene que ver con mostrar que los procesos de subjetivación, es decir, la constitución epocal de la subjetividad, es un efecto de la extensión primera y determinante de unas prácticas de ejercicio del poder que abordaremos desde la noción de "gobierno de la subjetividad". Como muestran los análisis históricos de Foucault (1996 y 2006), desde el siglo XVIII la noción de "gobierno" ha implicado la existencia de un conocimiento de lo que tiene que ser gobernado -infancia, familia, economía, sociedad-, en una situación concreta y en un momento determinado -tasas de mortalidad, tasas de productividad, tasas de desempleo-, al mismo tiempo que ha implicado la existencia de un conocimiento de los medios a través de los cuales se puede configurar, orientar y producir objetivos deseables en torno a aquello que se pretende gobernar. 
Algunos autores (Rose y Miller, 1992; Rose, 1997; Dean, 1999) proponen que la noción foucaultiana de "gobierno" nos remite a dos ámbitos de problematización, por un lado, al análisis de las racionalidades políticas, en tanto "trasfondo" en el cual se articula una determinada manera de concebir el poder; y por otro lado, al análisis de las tecnologías con las cuales el poder se materializa en una determinada situación histórica.

Con la noción de "racionalidad política”, nos referimos a la articulación de unas modalidades especificas de concebir el orden social, un marco que establece los límites de lo posible y lo imposible, al estilo de unos “juegos estratégicos de libertad” (Foucault, 2006). Como señala De Marinis (1999), cuando analizamos las racionalidades políticas nos podemos centrar en varios aspectos, entre los que podemos destacar dos: a) las diversas justificaciones morales de los modos de ejercer el poder por parte de las diferentes autoridades; y, b) las nociones sobre las formas apropiadas, los objetos y los límites de la propia acción política. En palabras del propio autor: "Las racionalidades políticas se enfrentan, dicho de un modo muy general, con la compleja temática de saber quién puede gobernar, qué es gobernar, qué o quién es gobernado” (De Marinis, 1999: 89). En nuestro caso, nos referimos a un campo discursivo donde lo social adquiere valor particular, donde los límites del bien común, la definición de los derechos y en definitiva los contenidos del imaginario de la ciudadanía, son fijados a través de una imagen de sujetos gobernantes y gobernados.

Por su parte, las “tecnologías de gobierno" corresponden a los dispositivos específicos que hacen circular el poder, que lo hacen actuar, modelar o dirigir en los diversos sistemas posibles (productivo, semiótico, identitario). Ya no se trata de los grandes esquemas racionalizadores del orden social, sino más bien, de los mecanismos prácticos y reales a través de las cuales las elites ejercen el poder. Como señalan acertadamente Rose y Miller: "If political rationalities render reality into the domain of thought (...) technologies of government seek to translate thought into the domain of reality, and to establish in the world of persons and things spaces and devices for acting upon those entities of which dream and scheme" (1992: 8)

Siguiendo esta propuesta, con la noción de "tecnologías de gobierno" pretendemos analizar aquellos instrumentos que materializan los discursos y prácticas de una racionalidad política específica, instrumentos diversos que articulan un saber experto en un ámbito de dominio particular. En el caso específico de este análisis, nos referimos a los productos del saber experto de los think tank que institucionalizan el conocimiento necesario para hacer funcionar la imagen de gobierno, gobernantes y gobernados.

De este modo, y a partir de estos dos ámbitos de problematización, podemos señalar que cuando hablamos del "gobierno de la subjetividad", nos estamos refiriendo a la acción de estas "racionalidades políticas" y sus diversas "tecnologías de gobierno". Estas prácticas de "gobierno" se constituyen en los discursos que en cada época establecen el control de los procesos de constitución de la subjetividad y la ciudadanía, actuando como 
fuerzas hegemónicas, fijando la identidad de los sujetos ciudadanos, sus atributos y espacios de actuación y llenando de significados su propia narración identitaria.

El sujeto ciudadano, en tanto "posición” llenada de significado por los discursos hegemónicos, está inevitablemente sujetado a estos procesos de control interno y externo que lo rigen. En efecto, como cualquier posición-sujeto, la ciudadanía es una producción de estas prácticas de poder que la constituyen como el lugar de re-significación política de la subjetividad, un lugar constitutivo y originalmente vacío, ya que: "Así como "yo" denota una posición en un conjunto de relaciones lingüísticas, una posición vacía que hace posibles mis enunciados únicos (...) "el ciudadano” también denota un lugar vacío” (Donald, 1999: 288).

Desde la perspectiva de Laclau (2000), este lugar vacío puede ser analizado como un significante particular que asume la función de representación de una totalidad de significados que resulta completamente inconmensurable con él, es decir, un significante que adquiere la capacidad de estar en lugar de todos los elementos que se articulan en la posición de un sujeto-ciudadano determinado. El punto que nos interesa de Laclau para nuestra argumentación, es que plantea una hipótesis explicativa sobre cómo es posible entender que el sujeto-ciudadano como lugar vacío sea llenado de significado, forma e identidad, a través de acciones de fuerza que, recuperando el legado Gramciano, denomina "hegemonía”. Al respecto Laclau y Mouffe nos señalan textualmente lo siguiente: "El campo general de emergencia de la hegemonía es el de las prácticas articulatorias, es decir, un campo en el que los "elementos" no han cristalizado en "momentos" (...) Es porque la hegemonía supone el carácter incompleto y abierto de los social, que sólo puede constituirse en un campo dominado por prácticas articulatorias" (1987: 155)

Podemos caracterizar la hegemonía como un tipo de relación por medio de la cual se constituye como totalidad "universalizada" una configuración ideológica, que para una identidad colectiva determinada, gobierna la producción del significado político de las acciones que se producen bajo su influencia. La relación hegemónica transforma las subjetividades implicadas en ella (es por tanto, aunque no sólo, un proceso de constitución y gobierno de subjetividades); no se refiere sólo al espacio público (empieza y tiene que ver con la vida cotidiana); ni es el resultado de una reconciliación humana definitiva (por ejemplo, como resultado de la desaparición de relaciones de poder). La relación hegemónica, por tanto, es constitutivamente política.

Para esta perspectiva (Laclau y Mouffe, 1987: 147-166), toda identidad aparece constituida en el punto de encuentro entre dos tipos de lógicas que participan en su constitución, las lógicas de la equivalencia y las lógicas de la diferencia, ambas incompatibles entre sí, pero absolutamente necesarias en el proceso de constitución de la subjetividad y la ciudadanía. Las lógicas de la equivalencia participan precisamente haciendo más simi- 
lares los elementos dispersos en un trasfondo a partir del proceso de exclusión radical de aquel elemento que niega todo el sistema de diferencias: su exterior constituyente (Laclau 2000). Es decir, el carácter antagónico de toda identidad supone que los elementos que quedan al interior del cierre que define su límite, se vuelvan equivalentes entre sí. Pero lo único que puede hacer equivalente entre sí a una dispersión de elementos diferentes, es una lógica de la diferencia. Entonces, ¿cómo se integran ambas lógicas en el proceso de la articulación de la identidad y la ciudadanía? Laclau y Mouffe dirían que es a partir de la capacidad que tienen los discursos para transformar un elemento particular en el representante de la equivalencia interna y la diferencia externa, un elemento significante que no posee un significado original y que más bien es llenado de él a partir de una acción hegemónica.

De este modo, hegemonía aquí quiere decir que una relación de poder concreta, en el marco de una racionalidad política que actúa como su trasfondo, llena de sentido un elemento particular para hacerlo significar representar- una dispersión de elementos que no están unidos por ninguna relación de necesidad, de ahí que para nuestra investigación fue relevante analizar los discursos del saber experto sobre la ciudadanía, como expresiones de las relaciones de poder que gobiernan los procesos de constitución de las propias representaciones de la ciudadanía.

El propio Laclau (2000) nos propone un ejemplo que a continuación parafraseamos de manera general. Después de la primera guerra mundial, en Italia, la gente decía con frecuencia que los fascistas habían sido capaces de llevar a cabo la revolución en la que los comunistas habían fracasado. Laclau se pregunta, ¿cómo fue posible que la gente pensara de este modo si los contenidos de ambos proyectos ideológicos eran tan radicalmente opuestos? La respuesta que propone el autor es simple: en la Italia de la época se percibía que el Estado que había surgido en el siglo XIX estaba en un proceso de rápida desintegración y que por tanto era necesaria una refundación radical del Estado Italiano, de modo que, el término "revolución”, pasó a significar esa refundación radical. De este modo, el término "revolución” paso a ser el elemento particular que permitió hacer equivalentes una serie de necesidades, deseos y proyectos en torno al límite común del discurso nacionalista de los fascistas, los cuales fueron capaces de hegemonizar la "revolución” al responder mejor a la amenaza de la desintegración nacional que actúa como un verdadero "exterior constituyente" en esta situación.

Como se deduce del ejemplo propuesto por Laclau, el tema fundamental en nuestro análisis son las racionalidades políticas en las cuales se articulan las representaciones de la ciudadanía a partir de un elemento hegemonizado. En el caso analizado por Laclau este elemento es "la revolución”, pero en nuestra investigación puede ser la democracia, el progreso, la libertad; el elemento que nos permitirá entender que sólo algunos significantes de los discursos disponibles se pueden articular en identidades ciudadanas, mientras que los demás se transforman en su exterior cons- 
titutivo, es decir, en sus condiciones de posibilidad o existencia. Por ello, las relaciones de poder implicadas en la noción de "gobierno", actúan sobre algunos elementos del discurso haciéndolos equivalentes en torno a una representación, un nombre, o una identidad y radicalmente diferente de aquello que se define como exterior. Si bien los ejemplos de Laclau son más bien macro-sociales y nos ilustran procesos discursivos de carácter histórico, el proceso de representación de los elementos a través de un significante particular, también opera a través de una materialización de las relaciones de una "microfísica del poder".

Esta distinción es relevante en nuestro análisis, toda vez que la noción de "gobierno de la subjetividad" que aquí proponemos responde a una forma de relación de poder que opera en el nivel de las condiciones de posibilidad y existencia del sujeto ciudadano, en el nivel de la hegemonía dirían Laclau y Mouffe, privilegiando la interfase en la cual, a partir de unas necesidades históricas se han ido constituyendo unos saberes expertos sobre la ciudadanía y el sujeto. El "gobierno de la subjetividad" opera en esta interfase en la cual se produce el proceso de articulación de las identidades ciudadanas en un lugar y momento histórico determinado.

De este modo, la noción de "gobierno de la subjetividad", se refiere a unas tecnologías de poder especificas que operan a través de los discursos hegemónicos que llenan de significado un elemento particular y lo transforman en la representación general en torno a la cual se articula una identidad llamada ciudadanía. A partir de este esquema conceptual, nos proponemos analizar concretamente en el caso Chileno, los procesos de construcción del sujeto-ciudadano que se producen en el saber experto de los think tanks seleccionados, como una manera de desplegar y confrontar este artefacto teórico con las vicisitudes de un proceso social e histórico especifico.

\section{Caso de investigación}

Como caso de investigación, se analizó la producción de tres centros de estudios de gran influencia política en Chile, a saber: el Instituto Libertad y Desarrollo (LyD), el Centro de Estudios Públicos (CEP) y la Fundación Chile 21 (Chile 21). Los tres think tanks seleccionados son organizaciones que producen sistemáticamente diversas tecnologías de conocimiento, tales como diagnósticos, encuestas de opinión pública, proyecciones económicas y múltiples documentos de trabajo con análisis críticos sobre políticas laborales, innovación, seguridad ciudadana, partidas presupuestarias o inversión pública. Específicamente, el análisis se realizó a partir de una lectura crítica de un conjunto de artículos divulgados en alguna de sus publicaciones regulares (documentos de trabajo, revistas y boletines) durante un período que se extiende entre el año 2003 y el año 2005, privilegiando especialmente este último año, por la posición estratégica que pasaron a tener los think tank estudiados, en la fundamentación de los programas de gobierno de los candidatos presidenciales de las elecciones del año 2005. 
Para la identificación del material de análisis se implementaron dos procedimientos sucesivos. En primer lugar, se construyeron categorías temáticas a partir de un muestreo intencionado por relevancia política (Stake, 2005). La definición de la relevancia de los temas propuestos para la búsqueda del material se realizó en función de una serie de entrevistas a informantes expertos quienes ponderaron el lugar de las temáticas en la realidad política chilena. Las categorías utilizadas finalmente en el levantamiento de la información fueron las siguientes: a) desigualdad y distribución del ingreso, b) superación de la pobreza, c) empleo/desempleo, d) calidad de la educación, e) desarrollo humano, y d) sociedad civil.

En segundo lugar, se realizó un proceso de reducción selectiva de los artículos identificados en la primera etapa, seleccionando textos específicos para cada categoría temática a través de un muestreo intencionado de variación máxima (Stake, 2005). Después de la aplicación de ambos procedimientos se obtuvo un corpus final de 42 documentos.

El plan de análisis se formuló en base a la combinación de herramientas y estrategias del análisis crítico del discurso provenientes tanto de sus versiones conversacionales (Wetherell y Potter, 1996), como de sus desarrollos orientados textualmente (Fairclough, 1992). Específicamente, nuestra propuesta de plan de análisis se propuso abordar el "contexto convencional" o plano del enunciado contenido en los textos, es decir, se planteó el objetivo de analizar la red de relaciones que se construyen entre las propuestas de los distintos Centros de Estudios en tanto representantes de una determinada tradición discursiva.

\section{Resultados}

El análisis del corpus textual identificó la existencia de dos discursos sobre el sujeto-ciudadano. Por un lado, el discurso post-ciudadano que materializa de manera explícita la racionalidad neoliberal y viene a representar el discurso hegemónico a la hora de definir las características del sujeto-ciudadano, sus procesos de integración social y sus espacios de actuación. Por otro lado, el discurso neo-ciudadano que representa un discurso subalterno que se propone incorporar elementos propios del imaginario de la ciudadanía social en la estructura discursiva de la racionalidad hegemónica, aportando de esta forma a su propia reproducción. A continuación presentamos brevemente ambos discursos.

\section{El discurso post-ciudadano}

El discurso "post-ciudadano" define a un sujeto que ha dejado atrás la representación tradicional de la ciudadanía social. Aquí el prefijo "post” representa superación, postergación y hasta cierto punto, negación de aquello que nos antecede y que emerge como la imagen de un tiempo pasado que ya 
no tiene ninguna vigencia para explicar la construcción de las identidades políticas en la sociedad actual.

El sujeto post-ciudadano es un sujeto libre y capaz de decidir racionalmente en el mundo, siendo responsable de las consecuencias positivas y negativas de sus decisiones, sus esfuerzos, sus iniciativas:

"La cultura del esfuerzo es dura. Los niños que tienen mayores dificultades tienen que esforzarse el doble para alcanzar los mismos resultados que los alumnos que tienen más facilidades. Sin embargo, uno de los factores que contribuyen a la formación de una buena autoestima es darse cuenta de que uno puede vencer los desafíos. Los profesores deben atreverse a exigir y deben resistir los reclamos de alumnos y padres. Tiene que haber conciencia de que el paternalismo y la sobreprotección son dañinos” (Eyzaguirre, 2004: 268).

La pauta de integración del sujeto post-ciudadano es la competencia. Nos referimos con este término a la capacidad, pero también a la obligación, que tiene el sujeto de ejecutar sus posibilidades de competir y ganar. Escuelas, profesores, trabajadores, jóvenes desempleados, todos deben competir para hacerse parte de aquel selecto grupo de sujetos posibles de ser llamados "ganadores":

"El mecanismo de libre elección tiene un gran potencial para generar calidad. Los padres se comportan como clientes, evalúan las diferentes ofertas y pueden "votar con los pies", retirando a sus hijos si sus demandas no son satisfechas (...) A los padres de niveles más pobres, se les da la oportunidad así de escapar de malos colegios y también de acceder a una oferta más diversificada en cuanto a valores o atención a sus necesidades especificas” (Fontaine, 2003: 4)

Es relevante señalar que este discurso, además, se define en contraposición a aquellos que niegan la competencia como pauta de convivencia hegemónica, a los cuales define como discursos incapaces para establecer una integración meritocrática al promover formas de integración que no incentivan a los sujetos a esforzarse para hacerlo mejor:

"Pero también la falta de dinamismo y calidad en el sector municipal, y en el país en general, están marcadas por el estatuto docente que protege exageradamente a los profesores municipales en el desempeño de sus funciones. La evaluación docente es un primer paso para ir eliminando los obstáculos que impone esta legislación a la formación de capital humano. Pero es claramente insuficiente. La urgencia en estos momentos es que los rendimientos académicos mediocres no se perpetúen en los establecimientos educativos del país. El estatuto docente y en general el actual marco institucional, aun con evaluación de por medio, no evita esa persistencia. Un fenómeno que en cualquier sistema educacional con estándares míni- 
mos de excelencia sería impensable” (Beyer, 2005: 89).

Desde este discurso, los límites para la integración social están definidos por las capacidades del sujeto para competir y sacar lo mejor de sí. Progresar es en primer lugar una cuestión de esfuerzo personal, de iniciativa, de capacidad individual. Estas nociones nos hablan de características específicas de un sujeto que resulta capaz de adaptarse a las condiciones cambiantes de un medio que le demanda permanentes operaciones sobre sí mismo para mantenerse vigente por sus propios méritos. De ahí la importancia de nociones como flexibilidad y capital humano para explicar el proceso de integración en ámbitos diversos como las relaciones laborales o la educación:

"La diferencia en las rentas del trabajo se explica principalmente por el stock de capital humano que tiene cada trabajador, donde se refleja tanto la escolaridad que alcanzó como la institución en donde se realizó” (LyD, 2005a: 3).

"La flexibilidad en horarios, contratos y sistema de remuneraciones también apunta hacia un mercado laboral más acorde con las necesidades de empresas y trabajadores, con prometedores efectos en participación laboral, empleo y productividad (Albagli, 2005:160).

Emerge el tema del capital humano de los sujetos como explicación fundamental de la desigualdad social. Se impone un sistema de representaciones de la integración social, ligado a la capacitación y la formación permanente, al mismo tiempo que el propio recurso humano se transforma en capital, móvil, adaptable y renovable:

"Hay que avanzar hacia un marco institucional distinto que ponga los aprendizajes y destrezas de los estudiantes en el centro de las consideraciones pedagógicas. Ello permitirá ir acumulando el capital humano necesario para seguir aumentando nuestro nivel de desarrollo y reducir las desigualdades que tanto preocupan (Beyer, 2005: 93).

“La desigualdad en la distribución de los ingresos, se vería afectada principalmente por la desigualdad salarial, que a su vez es explicada por la acumulación de capital humano, dejándole un mínimo poder explicativo al capital (LyD, 2005b:1)

El discurso post-ciudadano define un espacio de actuación para el sujeto en donde se expresa esta individualización y competitividad que le son intrínsecas. La metáfora que mejor representa los contornos de este espacio de actuación es una “carretera”, es decir, una suerte de espiral competitiva de integración a la cual convergen libremente todos quienes quieren ser parte de la forma de vida vigente. Nos referimos a un espacio en el cual se puede ejercer la capacidad y motivación que fundamenta nuestra propia libertad: 
"Lo importante acá es que no existan barreras a la entrada y que los actores en cualquier mercado puedan estar constantemente amenazados por la posibilidad de que otro sea más eficiente que él (...)” (Buchi, 2005: 16)

El espacio de integración del sujeto post-ciudadano es por definición competitivo y por extensión meritocrático. No es una carretera donde prime la igualdad social, sino la igualdad en el acceso individual a las oportunidades para poder competir, tal como lo ejemplifican los planteamientos liberales sobre la distribución del ingreso:

"cabe recalcar que lo que se busca no es una distribución del ingreso igualitaria per se, ya que las personas somos diferentes; lo que sí se busca es igualdad de oportunidades y una mayor movilidad social fundada en los méritos y las capacidades de las personas, para que todo quien se esfuerce tenga las posibilidades de estudiar o calificarse y de esa manera surgir por sus propios méritos (Castro y Sánchez, 2005: 41)

En este discurso, "igualdad de oportunidades" viene a representar una idea individualizada y competitiva de la integración social, de modo que significa algo así como la "eliminación de los obstáculos en el camino" de manera de asegurar que todos corran en igualdad de condiciones, porque la desigualdad se enfrentaría con libertad. Por ello, el sujeto post-ciudadano habita un escenario que estaría regido por unas reglas espontáneas que no deberían ser intervenidas por un principio externo al sistema, porque ello inevitablemente constituiría una arbitrariedad que distorsionaría el carácter natural del funcionamiento del propio sistema.

\section{El discurso del sujeto neo-ciudadano}

El discurso "neo-ciudadano" define a un sujeto que se propone actualizar y redefinir la representación tradicional de la ciudadanía social. Aquí el prefijo "neo” representa recuperación, renovación, actualización, expresando un esfuerzo por reconstruir un imaginario que si bien se evalúa como históricamente agotado, se aspira a reformularlo en función de las nuevas necesidades de la sociedad contemporánea.

El problema central de este discurso es la asociatividad material y/o simbólica que permite que el sujeto se constituya en individuo-ciudadano, de modo que su propuesta es que la red de vínculos sociales constituiría un elemento contextual que asiste al sujeto en su proceso de transformación en un individuo-ciudadano propiamente tal:

"para existir como individuo es necesario tener los soportes, y por tanto, interrogarse sobre aquello que hay tras él para permitirle existir como tal. En este sentido, no hay posibilidad alguna de un individuo autónomo, propietario de sí mismo si no posee los bienes míni- 
mos que lo ponen fuera de situaciones de dependencia y sumisión” (Márquez, 2005: 12).

Para el discurso neo-ciudadano, el proceso de integración social estaría representado por la formación de vínculos sociales. A diferencia del discurso post-ciudadano, este discurso asume que si bien los procesos de competencia individual son centrales en el proceso de integración, las redes de apoyo estatales o de la propia sociedad civil, constituyen elementos igualmente cruciales a la hora de explicar los procesos de integración, proponiendo una suerte de alianza entre Estado y Sociedad civil:

"Nuestro planteamiento apunta a establecer que existen campos de confluencia posible entre políticas públicas y sociedad civil en una perspectiva democratizadora” (De la Maza, 2005: 17)

Desde esta perspectiva, el proceso básico que explica la integración del sujeto neo-ciudadano es la acumulación a través de su relación con los otros, de las capacidades personales y sociales necesarias para enfrentar de mejor manera los requerimientos del mundo social:

"En el plano social el desafío es de acrecentar la capacidad de concertación entre actores y el establecimiento de puentes que aminoren la fragmentación social y faciliten las acciones colectivas de bien público en contextos de diversidad social” (De la Maza, 2005: 16)

El discurso neo-ciudadano articula el imaginario de la solidaridad y la igualdad en su proyecto de promoción de la equidad a través de una red de políticas sociales que actúan como los soportes materiales de los sujetos que viven en riesgo social, especialmente a través de la constitución del Estado en una suerte de garante social:

"Tal como se ha demostrado en estos años, el sólo crecimiento no asegura una generación de empleos suficientes para absorber una fuerza de trabajo en expansión y se requiere de políticas públicas pro activas a favor de la generación de nuevos empleos de calidad (Chile 21, 2005: 5).

Para este discurso, las políticas sociales vienen a constituir la expresión institucional de una red de vínculos que promueven una forma de igualdad, pero a partir de un principio ético más que político:

"Ellas constituyen una de las expresiones "pasivas” del vínculo social y a su manera, dan testimonio de una forma de igualdad. Esta dimensión simbólica es esencial y participa del principio mismo de la solidaridad. Para que ello ocurra es imprescindible sin embargo, la existencia de una asistencia no degradante, que asuma la forma de un derecho y no de un tutelaje. Cualesquiera que sean sus dificultades, el individuo pretende siempre seguir siendo un ciudadano." (Márquez, 2005: 15-16). 
En este discurso los vínculos sociales se transforman en una verdadera forma de capital, de modo que la red social y la red institucional de políticas sociales vienen a representar materialmente el grado de capital social acumulado por los sujetos. De modo análogo a la noción de capital humano, la idea de capital social se utiliza para destacar el aporte creativo de determinadas formas de organización, en la estabilidad del propio modelo económico:

"La combinación de crecimiento económico con desarrollo social, es decir, una economía que se pone al servicio del hombre y que construye capital social no se produce espontáneamente, sino que es la consecuencia de una política deliberada, de una cierta propuesta económico-social dirimida democráticamente y que responde a un cierto proyecto de país” (Hardy, 2004: 9).

Agregando más adelante:

"aquellos países que descuidan su capital humano y no construyen capital social, dejan de crecer aún si tienen condiciones materiales ventajosas y aún si existe un clima mundial económicamente propicio” (Hardy, 2004: 10)

El discurso neo-ciudadano define un espacio de actuación para el sujeto en el cual se expresa la asociatividad y la solidaridad que le son intrínsecos. La metáfora que mejor representa los contornos de este espacio de actuación es una "red” de vínculos y relaciones que sostiene a todos aquellos que resbalan en la inestable tarea de integrarse en la forma de vida vigente. Es decir, podemos representar visualmente este escenario de vínculos y relaciones como una "red de seguridad" que se ubica bajo la espiral de la integración para poder recibir y proteger a todos aquellos que caen de ella o a aquellos que definitivamente nunca han logrado estar en la espiral:

"un Sistema de Protección Social que, a partir de la experiencia del Chile Solidario, logre ampliarse y extenderse para proteger a quienes, no siendo extremadamente pobres o habiendo superado la pobreza, por razones circunstanciales quedan socialmente desprotegidos y en condiciones de riesgo social. Este nuevo Sistema de Protección Social, que requiere una nueva institucionalidad social, acoge la idea de que, si bien es necesario seguir apoyando el esfuerzo focalizado hacia los más pobres, la noción de derechos universales obliga al Estado a proteger socialmente a todos los ciudadanos que lo requieran" (Chile 21, 2005: 3)

El discurso neo-ciudadano restituye, de este modo, el imaginario de la ciudadanía a través de la implementación de esta red de políticas sociales que protege a los más débiles e incentiva a los más capaces para integrarse a una sociedad profundamente desigual. Emerge de este modo, El 
imaginario de la "igualdad de oportunidades” aparece aquí explicando el proceso de apoyo a la integración social y proponiendo una alternativa a la hegemonía del discurso del mérito personal como único criterio de integración, ya que para el discurso neo-ciudadano considerar sólo el mérito personal como factor de movilidad social, generaría inevitablemente movilidad descendente al reproducir las desigualdades en las oportunidades de origen.

Por ello, para el discurso neo-ciudadano no sólo habría que asegurar condiciones de equidad para el sujeto (igualdad de entrada en el sistema), sino también unos niveles de igualdad en su sentido amplio (igualdad en los resultados):

"Lo claro es que el sólo crecimiento no asegura solucionar el tema de las desigualdades y más bien amenaza con acrecentarlo si no va acompañado de activas políticas públicas a favor de una mayor igualdad de oportunidades y de resultados" (Contreras, 2004: 4)

"los desafíos de un futuro gobierno no sólo está asegurar igualdad de oportunidades (en el origen), sino que garantizar también determinados resultados o desenlaces. Esta orientación apunta a una concepción central de una propuesta progresista, la igualdad de derechos, en que la equidad es parte de un bien mayor, la igualdad, basada en la noción de que por el solo hecho de ser ciudadanos somos sujetos de derecho" (Chile 21, 2005: 2).

La dialéctica entre igualdad de oportunidades e igualdad de resultados nos remite necesariamente al problema de la "igualdad redistributiva”. Desde este discurso el sujeto neo-ciudadano debe ser "incentivado" a integrarse por sus propios medios en una clave profundamente liberal, pero también debe ser "protegido" cuando no logra la tarea de integrarse a partir de un registro profundamente social, demostrando sin embargo, que la protección social queda supedita a los resultados del esfuerzo individual.

\section{Discursos ciudadanos y contrariedad subalterna}

A partir de la presentación de ambos discursos podemos abordar el problema del tipo de relación que establecen entre sí. Al respecto, nuestra propuesta es que los discursos post y neo-ciudadano establecen una relación de "contrariedad subalterna” ya que ambos establecen una relación de diferenciación parcial entre algunos de sus elementos específicos, pero actuando en el marco de la hegemonía de una racionalidad política común. 


\section{Esquema $\mathbf{N}^{\circ} 1$ \\ Representación de la relación entre los discursos ciudadanos}

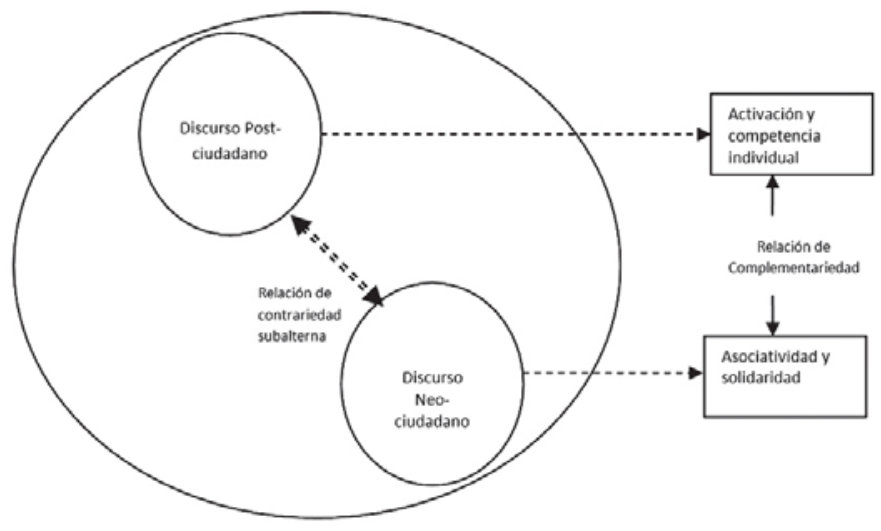

Como muestra el esquema $N^{0} 1$, proponemos que los discursos post y neo-ciudadano se articulan "escalonadamente", tal que cada uno de ellos explica parcialmente una fase del proceso de integración/desintegración del sujeto-ciudadano. Por un lado, a partir del discurso del mérito y el esfuerzo personal se estructura una fase de la integración como activación individual para la competencia; mientras que por otro lado, a partir del discurso de la solidaridad y la asociatividad se estructura una fase de la integración como protección y contención social.

Para ilustrar esta relación consideremos brevemente tres aspectos específicos de las relaciones de contrariedad que se establecen entre los discursos post y neo-ciudadano. Estos tres puntos de debate o contrariedad son los siguientes: a) el problema de la relación entre igualdad y libertad y sus implicancias sobre el problema de la individualidad; b) el debate entre la competencia y la asociatividad como principio de integración social; y finalmente, c) la dialéctica entre la igualdad de oportunidades y la igualdad de oportunidades más igualdad de resultados.

En primer lugar, tenemos la tensión entre la representación del individuo libre versus la del individuo asociado y el debate en torno a la libertad y la igualdad vinculado a ambas representaciones. Efectivamente, ambos discursos postulan visiones diferentes sobre el sujeto y su relación con la libertad y la igualdad. Así, el discurso post-ciudadano enfatiza el carácter central de la individualidad como fundamento de la libertad negativa y ésta como justificación de la responsabilidad individual, mientras que el discurso neo-ciudadano subraya la naturaleza relacional de un sujeto que necesitaría de los soportes sociales para alcanzar la libertad positiva como autonomía. 
Sin embargo, en la integración de los discursos sobre el sujetociudadano la tensión entre el ideario de la libertad y la igualdad se resuelve a favor del primero. En ambos discursos la libertad es el fin último que moviliza el proceso social, de modo que la igualdad constituye un valor subalterno, una estrategia que sólo restituye la autonomía a aquellos que por sus propios medios no pueden ejercerla.

En segundo lugar, tenemos la tensión que se establece entre "capital humano y flexibilidad” versus "capital social y solidaridad”. A pesar de la aparente diferencia entre estos discursos, ambos apelan a la misma metáfora de la "capitalización”. En un caso, lo que se capitaliza son competencias individuales en el marco de una trayectoria de formación y/o capacitación; mientras que en el otro, lo que se acumula son relaciones y vínculos sociales en el marco de experiencias de solidaridad y asociatividad.

El punto es que ambos discursos convergen en una idea fuerza que cuestiona el principio de la solidaridad orgánica del sistema social, imponiendo una versión cada vez más contractual de la propia ciudadanía como actividad "empresarial”. La asociación con otros constituye una estrategia distinta, pero complementaria a las estrategias individuales de acumulación de habilidades personales, respondiendo ambos tipos de "empresas" a la misma estrategia hegemónica de inversión o acumulación de capital y jugando ambas un papel estratégico en la postergación del conflicto y el antagonismo social.

En tercer lugar, tenemos la tensión entre el discurso de la igualdad de oportunidades versus el discurso de la igualación de oportunidades más igualdad de resultados. El debate entre el discurso post y neociudadano está centrado en cómo significar la igualdad de oportunidades, en el primer caso representa una materialización de la "libertad negativa” a través de la limitación de la acción de fuerzas externas al individuo que impidan el ejercicio de sus capacidades, mientras que en el segundo caso, la igualdad de oportunidades en la entrada del sistema debería estar acompañada de intervenciones de un Estado social que asegure unos niveles mínimos de resultados, como una versión básica de los derechos universales de ciudadanía social.

Sin embargo, ambos discursos establecen una relación de complementariedad y en ningún caso de antagonismo, al definir como punto de consenso entre ambos, una versión liberal de la igualación de las oportunidades de entrada y dejando el discurso de la igualdad de resultados como un planteamiento subalterno, que se conecta más con el imaginario de la caridad y el control de la desviación, que con el imaginario de la ciudadanía social. 


\section{Discusión}

Los discursos post y neo-ciudadanos analizados en este trabajo establecen relaciones de contrariedad en distintos niveles, las cuales se integran en una estructura discursiva común al formar parte ambos discursos de una misma racionalidad política. Individualidad-sociabilidad, capital humano-capital social, igualdad para emprender-igualdad para pertenecer, constituyen las contrariedades específicas que se pueden identificar entre ambos discursos, las cuales sin embargo, tal como ilustra el esquema $\mathrm{N}^{\circ} 2$, no constituyen un antagonismo suficiente como para romper la unidad del sistema discursivo sobre la ciudadanía, y más bien, se integran en un único sistema articulado a partir de una racionalidad política fundante.

\section{Esquema 2}

Síntesis del análisis de los discursos sobre los sujetos-ciudadanos y sus condiciones de posibilidad

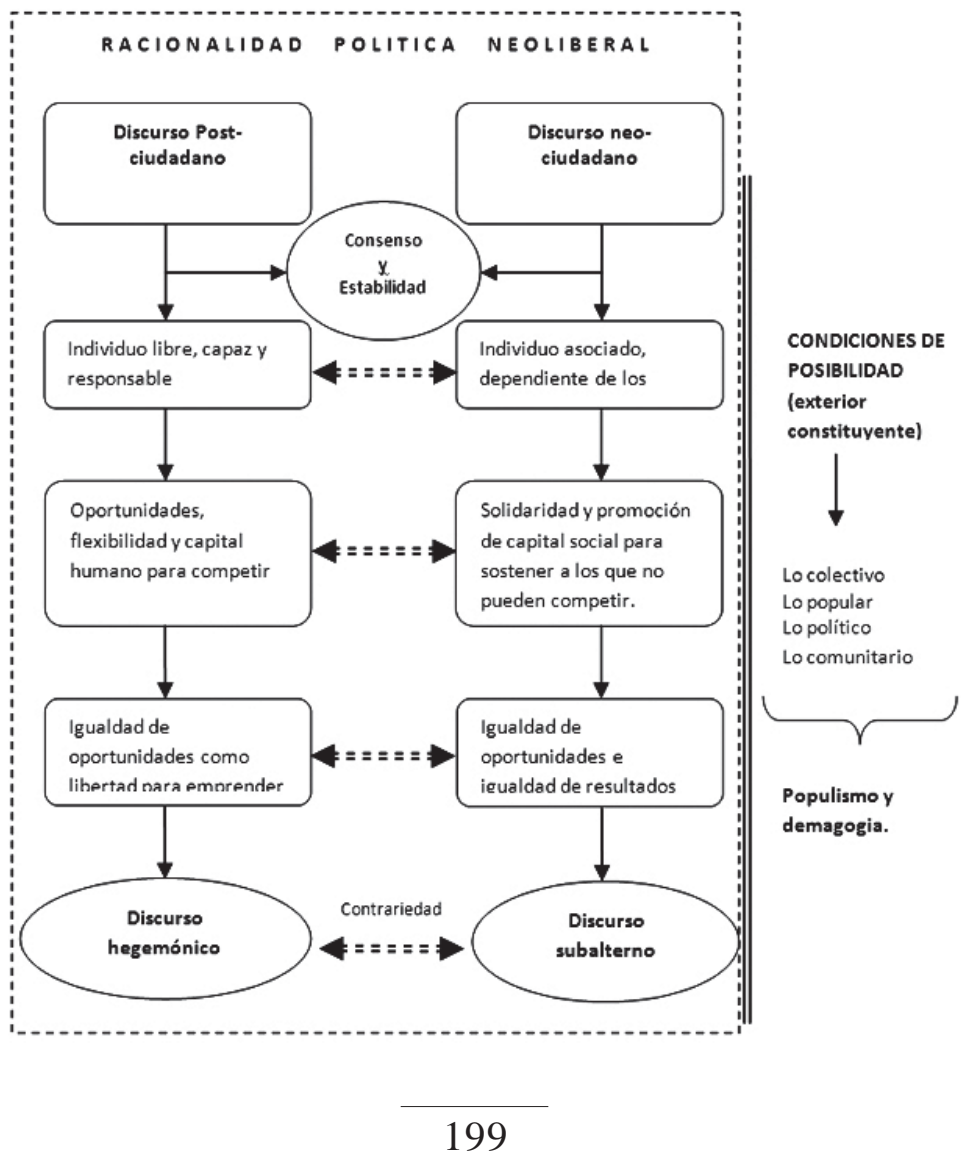


La unidad de este sistema discursivo se sostiene en la expulsión de todo contenido que ponga en cuestión su racionalidad fundante, transformando a estos contenidos expulsados del sistema, en las condiciones de posibilidad de la hegemonía de esta misma racionalidad. En nuestro análisis de discursos de los think tank, varios significantes tales como popular, colectivo, político, comunitario, actúan como los nombres de aquellos elementos expulsados del sistema discursivo sobre la ciudadanía, de modo que a partir de su lugar de exterioridad establecen las condiciones de posibilidad para que los discursos post y neo-ciudadanos se transformen en partes de una misma racionalidad política que aparece como hegemónica.

Nuestra propuesta es que el significante que permite articular las lógicas de la equivalencia y la diferencia, es decir, que permite que los discursos post y neo-ciudadano se hagan equivalentes entre sí al mismo tiempo que se diferencien de manera radical de los contenidos expulsados del sistema discursivo, es la naturalización del "consenso y la estabilidad" como una necesidad constitutiva de lo social. Esto significa que las representaciones post y neo-ciudadanas se construyen dentro del límite que establece esta tendencia por privilegiar el consenso y la estabilidad por sobre otros elementos, expulsando a una posición de exterioridad del sistema a todos aquellos significantes que ponen en riesgo dicha necesidad fundante.

En nuestro caso de estudio, por ejemplo, el discurso post-ciudadano utiliza el término "populismo" y el discurso neo-ciudadano el término "demagogia” para referirse a los proyectos políticos que subvierten el orden vigente, poniendo en acción la tendencia por privilegiar la estabilidad y excluir el disenso. En estos ejemplos, los significantes "estabilidad y consenso" pasan a cumplir la función de hacer equivalentes entre sí a los discursos post y neo-ciudadanos y hacer a ambos diferentes de todo lo que no representa este orden y al cual se le excluye como populismo y demagogia. Por ello es que todo discurso que apela a lo popular o lo colectivo se constituye en un proyecto imposible para el saber experto, porque la necesidad histórica que está en falta y que se pone en juego en su acción hegemónica, es precisamente la "estabilidad y el consenso".

Sin embargo, este proceso de expulsión del disenso de las representaciones de la ciudadanía que identificamos en los discursos del saber experto, hace parte de un proceso más general de despolitización de lo social. Manifestaciones de este proceso las podemos observar en la crisis sistemática de las vías formales de participación política que se vienen agudizando cada día más desde principios de la década de los noventa (Baño, 2011), pero también en la tendencia general a descolectivizar la participación social en una suerte de "psicologización" de la asociatividad a través de tecnologías como el voluntariado y los fondos concursables (Sandoval y Hatibovic, 2010). Esta última tendencia resulta particularmente relevante para nuestro análisis, pues constituye un ejemplo del modo como el sistema discursivo hegemónico resignifica permanentemente términos como participación social, con el objeto de mantener la exclusión de aquellos elementos simbólicos que subvierten la unidad del sistema discursivo. 
En efecto, en los discursos de los think tanks analizados, la ciudadanía se institucionaliza en las acciones tácticas del Estado y/o el mercado a través de tecnologías como los proyectos concursables, las asociaciones voluntarias, y en las versiones más radicales, a través del propio consumo. Los sujetos-ciudadanos participan a través de las vías que la propia racionalidad hegemónica establece como formas de acción legal, de modo que por ejemplo, en los discursos post-ciudadanos la participación social y política se reduce a la asociación táctica entre sujetos individualmente libres; mientras que en los discursos neo-ciudadanos la participación responde a la asociación entre sujetos que habitan una red de vínculos sociales. En ambos casos, la participación que se propone es acción controlada, funcional, organización sistémica, en definitiva, la participación se inscribe dentro de los márgenes del "consenso y la estabilidad”.

Podemos concluir que en los discursos expertos analizados en este trabajo se articula una estrategia de gobierno de la subjetividad que podemos llamar "psicologización económica de la ciudadanía”, estrategia a partir de la cual los problemas políticos se transforman en problemas de "gestión psicosocial”. Como afirman Crespo, Revilla y Serrano (2009: 99) “... la psicologización produce una responsabilización del sujeto sobre su situación, buena o mala, que corre pareja de una naturalización del estatus quo que consideraría que lo real es moral, esto es, que cada uno tiene lo que se merece, pues es fruto de sus acciones individuales y no de constricciones estructurales, sociales. Esta psicologización produce una despolitización de la realidad social, con la consiguiente desaparición de los conflictos sociales".

Esta forma de "gobierno de la subjetividad" se propone controlar el orden social y psicológico que se articula en los márgenes de los discursos sobre la ciudadanía. No obstante, como señalaba el propio Foucault (1996; 2006), dicho control no opera a través de prácticas prohibitivas sino productivas, no se propone la destrucción explícita de un orden político sino la producción implícita de un orden psicológico. Nos referimos a una estrategia de gobierno que recorta nombres y memorias, al mismo tiempo que reproduce movimientos y deseos, produciendo prácticas que actúan sobre los procesos de retroalimentación que permiten que un sujeto se gobierne a sí mismo (Frade, 2007; Kiersey, 2009; McLaughlin, 2010). Un ejemplo lo podemos encontrar en el discurso de la empleabilidad y la activación laboral, ambos discursos transforman un problema social como el desempleo en una temática psicosocial, donde el problema pasa a ser el mejoramiento de las capacidades individuales del sujeto para que se convierta en una oferta atractiva para el mercado laboral (Sandoval, 2009; Crespo y Serrano, 2010).

Esta estrategia de "gobierno de la subjetividad" se articula entre ambos discursos, porque las posiciones de sujeto-ciudadano que identificamos en este análisis tienen el mandato de aprender a capitalizar sus oportunidades para enfrentar los problemas sociales, sean estas oportunidades intrínsecamente individuales como en el caso del capital humano y la 
empleabilidad del discurso post-ciudadano, o tácticamente sociales como en el caso del capital social y las asociaciones voluntarias del discurso neociudadano. En ambas representaciones de la ciudadanía, la política deviene en gestión del sí mismo y de las relaciones que establecemos con los demás, demandando un proceso de manipulación permanente de los recursos personales y relacionales para lograr cumplir la tarea de transformar lo humano y lo social en capital.

Finalmente, podemos afirmar que los discursos del saber experto que analizamos en este trabajo construyen una ciudadanía que es pura subjetividad sin política, es decir, una representación pospolítica del sujeto ciudadano, donde su campo de actuación queda definido como un "terreno neutral en el cual diferentes grupos compiten para ocupar las posiciones de poder y cuyo objetivo sólo es "desalojar" a otros para ocupar su lugar, sin poner en cuestión la hegemonía dominante ni transformar profundamente las relaciones de poder” (Mouffe, 2005: 87). Podríamos decir que la metáfora del "desalojo" además de representar la ciudadanía que se construye en el sistema discusivo hegemónico, también expresa de buena forma la competencia sin antagonismo que se establece entre los discursos de los think tanks estudiados en este trabajo, entre los cuales solo se emprende una disputa por administrar la política, mientras todos adhieren con grados diversos de entusiasmo, al consenso en torno al sistema que la política debe administrar. 


\section{Bibliografía}

Albagli, Elías (2005), "Mercado laboral y crecimiento económico. Recomendaciones de política para Chile”. Estudios Públicos No 99. [CEP]

Beyer, Harald (2005), "Productividad, desigualdad y capital humano: Los complejos desafíos de Chile”. Estudios Públicos Nº 97. [CEP]

Buchi, Hernán (2005), "La desigualdad se combate con más libertad”. Revista Lyd $\mathrm{N}^{\circ}$ 158. [LyD]

Castro, R. y Sánchez R. (2005), “Una nueva mirada a la distribución del ingreso en Chile”. Serie Informe Social No 91. [LyD]

Chile 21 (2005), "Plan de igualdad. Igualdad de oportunidades y de resultados”. Fundación Chile 21, Santiago. [Chile 21]

Contreras, Marcelo (2004), "Enfrentar las desigualdades: ¿Un Consenso Nacional?”. Coyuntura, Fundación Chile 21. [Chile 21]

Crespo, Eduardo y Serrano, Amparo (2010), “The Psychologisation of Work the Deregulation of Work and the Governmentof Will". Annual Review of Critical Psychology, $\mathrm{N}^{\circ}$ 8, pp. 43-61. Recuperado el 25 de abril de 2011 desde: www.discourseunit.com/arcp/8.htm

Crespo, Eduardo, Revilla, Carlos y Serrano, Amparo (2009), "Del gobierno del trabajo al gobierno de las voluntades: el caso de la activación". Psicoperspectivas, VIII (2), 82-101. Recuperado el 05 de octubre de 2010 desde http://www.psicoperspectivas.cl

De la Maza, Gonzalo (2005), "Sociedad civil, iniciativas locales y desafíos para las Políticas Públicas”. Colección Ideas Año 5 Nº 54, Junio 2005. [Chile 21]

De Marinis, Pablo (1999), “Gobierno, gubernamentalidad, Foucault y los anglofoucaultianos (O un ensayo sobre la racionalidad política del neoliberalismo). En: Ramos, Ramón y García Selgas, Fernando (ed.) Globalización, riesgo y reflexividad. Tres temas de la teoría social contemporánea. CIS, Madrid.

Dean, Mitchell (1999), Governmentality. Power and rule in modern Society. Sage, London.

Donald, James (2003) "El ciudadano y el hombre de mundo". En: Hall, Stuart y Du Gay, Paul (comps.) Cuestiones de identidad cultural. Amorrortu, Buenos Aires. [1996]

Eyzaguirre, Bárbara (2004), “Claves para la educación en pobreza”. Estudios Públicos $\mathrm{N}^{\circ}$ 93. [CEP] 
Fairclough, Norman (1992) Discourse and social change. Polity Press, Cambridge.

Fontaine, Loreto (2003), “Cuotas Obligatorias de Alumnos "Vulnerables” en la educación subvencionada: Antecedentes para la discusión”. Punto de referencia $\mathrm{N}^{\circ}$ 267. [CEP]

Foucault, Michel (2006), Nacimiento de la biopolítica. Curso en el collage de France (1978-1979). Fondo de Cultura Económica, Buenos Aires. [1978]

Ídem (1996), Las tecnologías del yo. Paidós, Barcelona.

Frade, Carlos (2007) “Gobernar a otros y gobernarse a sí mismo según la razón política liberal”. En: REIS, 119, 35-69.

Gárate, Manuel (2008), “Think Tanks y Centros de Estudio. Los nuevos mecanismos de influencia política en el Chile post-autoritario”, Nuevo Mundo Mundos Nuevos, Coloquios, 2008. Recuperado el 17 de mayo de 2011 desde: http://nuevomundo.revues.org/11152.

Garcé, Adolfo (2009), “Panorámica de la relación entre Think Tank y Partidos Políticos en América Latina”. Extracted from Thinking Politics: Think Tanks and Political Parties in Latin America (Spanish): International Institute for Democracy and Electoral Assistance, Stockholm, Sweden.

Grondona, Ana Lucía (2010), "Políticas sociales en Argentina: De tecnócratas y Pastores”. En: Cassigoli, Isabel y Sobarzo, Mario (Comp.), Biopolíticas del Sur. Editorial ARCIS, Santiago de Chile.

Hardy, Clarisa (2004), “Algunas reflexiones sobre la relación entre políticas económicas y sociales”. Colección Ideas Año 5, No 39. Enero 2004. [Chile 21]

Kiersey, Nicholas (2009), “Neoliberal Political Economy and the Subjectivity of Crisis: Why Governmentality is Not Hollow”. Global Society, Vol. 23, No. 4, 363-386.

Laclau, Ernesto (2000), Nuevas reflexiones sobre la revolución de nuestro tiempo. Nueva Visión, Buenos Aires. [1993].

Laclau, Ernesto y Mouffe, Chantal (1987), Hegemonía y Estrategia Socialista. Hacia una política Democrática Radical. Siglo XXI, Madrid. [1985].

Lyd (2005a), “Evaluación del proceso de privatización en Chile”. Temas Públicos No 709, 14 de Enero del 2005. [LyD]

Lyd (2005b), “Cómo las privatizaciones mejoraron la distribución del ingreso”. Temas Públicos N 735, 12 de Agosto del 2005. [LyD] 
Márquez, Francisca (2005), “Por una política social de respeto”. Colección Ideas $\mathrm{N}^{\circ}$ 52, Abril 2005. [Chile 21].

McGann, James G. (2007), Think tanks and policy advice in the US: academics, advisors and advocates. Routledge, London; New York, NY.

McLaughlin, Kenneth (2010), "Psychologisation and the Construction of the political subject as vulnerable object”. Annual Review of Critical Psychology, $\mathrm{N}^{\circ}$ 8, pp. 63-79. Recuperado el 25 de marzo de 2011 desde www.discourseunit.com/arcp/8.htm

Miller, Peter y Rose, Nikolas (1990), “Governing economic life”. En: Economy and Society $N^{\circ} 19,1-13$.

Moreno, Marco (2010), Think Tank en Chile: Estilos y prácticas tecnocráticas en la formación de políticas. Revista Enfoques Vol. VIII, Núm.12: 103-125.

Mouffe, Chantal (2005), "Política y pasiones: las apuestas de la democracia”. En: Arfuch, Leonor (Comp.) Pensar este tiempo. Espacios, afectos, pertenencias. Paidós, Buenos Aires.

Pautz, Hartwig (2010), “Think Tanks in the United Kingdom and Germany: Actors in the Modernisation of Social Democracy”. The British Journal of Politic and International Relations. Vol. 12 (2): 274-294

Rose, Nikolas (1997), "El gobierno en las democracias liberales "avanzadas”: del liberalismo al neoliberalismo”. Archipiélago, Cuadernos de crítica de la cultura $\mathrm{N}^{\circ}$ 29: 25-44.

Rose, Nikolas y Miller, Peter (1992), "Political power beyond the state: problematics of government”. British Journal of Sociology (43) 2, 173205.

Sandoval, Juan (2009), "Representaciones de la ciudadanía en los discursos del 'saber experto': La individualización de la desigualdad”. Psicoperspectivas, VIII (2), 35-56. Recuperado el 10 de junio de 2010 desde: http://www.psicoperspectivas.cl

Sandoval, Juan y Hatibovic, Fuad (2010), “Socialización política y juventud: el caso de las trayectorias ciudadanas de los estudiantes universitarios de la región de Valparaíso”. Ultima Década Vol.18, N³2: 11-36.

Stake, Robert (2005), “Qualitative Case Studies”. En: Denzin, Norman y Lincoln, Yvonna (Ed.) Handbook of Qualitative Research. Thousand Oaks: Sage Publications.

Weidenbaum, Murray (2010), "Measuring the Influence of Think Tanks". Social Science and Public Policy N47:134-137. 
Polis, Revista de la Universidad Bolivariana, Volumen 11, $N^{\circ}$ 32, 2012

Wetherell, Margaret y Potter, Jonathan (1996), "El análisis del discurso y la identificación de los repertorios interpretativos”. En: Gordo, Ángel y Linaza, José Luís (Comp.) Psicologías, discursos y poder (PDP). Madrid, Visor.

Recibido: 03.12.2011

Aceptado: 25.07.2012 